\title{
Effect of Long Term-Administration with Gymnema sylvestre R. BR on Plasma and Liver Lipid in Rats
}

\author{
Norihiro Shigematsu, ${ }^{*, a}$ Ryuji Asano, ${ }^{b}$ Makoto Shimosaka, ${ }^{c}$ and Mitsuo OKazaki ${ }^{d}$ \\ Biosci. Textile Technol., Shinshu University, ${ }^{a}$ 3-15-1 Tokida, Ueda, Nagano 386-8567, Japan, Dept. Verterinary \\ Pharmacol., College of Bioresource Sci., Nihon University, 1866 Kameino, Fujisawa, Kanagawa 252-8510, Japan, Dept. \\ Applied Biol., Shinshu University, ${ }^{c}$ 3-15-1 Tokida, Ueda, Nagano 386-8567, Japan, and Gene Research Center, Shinshu \\ University, ${ }^{d}$ 3-15-1 Tokida, Ueda, Nagano 386-8567, Japan. Received November 16, 2000; accepted February 23, 2001
}

\begin{abstract}
Extract of Gymnema sylvestre leaves was administered to rats receiving either a high fat diet or normal fat diet for 10 weeks to investigate its influence on plasma and liver lipids and on visceral fat accumulation. In addition, its effect was compared with those of chitosan and the influence of combined use of these two substances was also evaluated. Within the high fat diet groups, the extract suppressed body weight gain and accumulation of liver lipids to the same extent as chitosan and the combined use. In addition, intraperitoneal fat and fat drop vacuoles on the epithelium of renal tubules, noted in the high fat diet group, were scattered by administration of the extract with the same results as for chitosan and combined use. Within the normal fat diet groups, plasma triglyceride levels decreased by administration of the extract, with similar results as chitosan and combined use. Concerning plasma total cholesterol, there was no decreasing effects with the extract, as found with chitosan and combined use. However, the effect of chitosan on plasma total cholesterol tended to be enhanced when used in combination with the extract. In addition, long-term administration of the extract did not show any influence on hematological and blood chemical parameters.
\end{abstract}

Key words Gymnema sylvestre; chitosan; plasma lipid; liver lipid

Gymnema sylvestre R. BR. is a wild plant classified in the Asclepiadaceae family and is widely distributed in Southern India, tropical Africa and Australia. It has been used in Indian traditional medicine, "Ayurvedic medicine," from ancient times and is said to be effective in promoting urination and digestion, tonifying and improving diabetes. ${ }^{1,2)}$ Its suppressive effects on sweetness are well known. ${ }^{3)}$ In Japan, $G$. sylvestre has been widely used as a health food in tea bags, tablets, beverages and confectioneries in recent years. There has been a lot of research on its involvement in carbohydrate metabolism from the viewpoint of nutrition and food science or pharmacognosy. ${ }^{4-6)}$ Various effects have been reported, such as suppression of glucose absorption in the small intestine of rats, reduction of plasma glucose increment in the oral sucrose tolerance test, significantly lowered blood glucose and insulin values in dogs as well as suppression of insulin increase in glucose tolerance tests in men and the alleviation of diabetic symptoms in patients with non-insulin-dependent diabetes mellitus. ${ }^{7,8)}$ As for the active substances involved in G. sylvestre, the triterpenoid saponin and its derivatives have been identified. These are glycosides where gymnemagenin is formed by attachment of glucronic acid to the triterpenoid structure as aglycone. This glycoside and its derivatives are referred to as gymnemic acids. ${ }^{9)}$ Other than these glycosides, conduritol A with a tetrahydroxyhexene structure has been also confirmed to be involved in glucose absorption. Moreover, the peptide grumarin, which is a peptide consisting of 35 amino acids, has been shown to be involved in suppression of sweetness. ${ }^{10)}$ However, there are fewer reports dealing with its influence on lipid metabolism compared to those on carbohydrate metabolism. Findings concerning the lipid metabolism of G. sylvestre thus far reported are as follows: (1) although it slightly decreased serum cholesterol and triglyceride values in obese rats fed on high carbohydrate and low fat diets, there were no effects observed in emaciated rats; (2) intraperitoneal injection of gymnemic acid had no influence on blood cholesterol, triglyceride or free fatty acid ${ }^{4)}$; (3) when the extract of G. sylvestre leaves, highly concentrated with gymnemic acid, was administered at high dose levels, fecal excretion of steroids showed a dose-related increase. ${ }^{11}$ All these findings were obtained under limitative conditions. The aims of this current study were to investigate the longterm influence of G. sylvestre on lipid metabolism in rats fed a high fat diet, and to compare with the effects of chitosan, ${ }^{12,13)}$ a substance known to be effective in lowering cholesterol levels. In addition, the effects of these treatments on rats with a normal fat diet were investigated in the same way. This experiment was performed according to the Experimental Animal Regulations (IEXAS).

\section{MATERIALS AND METHODS}

Materials The feed stuff CE-2 (product of Nihon Crea Co., Ltd.) was used as normal fat diet (NF), and a modified feed stuff was used as a high fat diet (HF), in which beef tallow was added to CE- 2 to the extent of $20 \%$ by weight. The nutritive constituents of the respective diets were as follows; water content (NF: $8.8 \%$, HF: $7.0 \%$ ), protein (NF: $25.2 \%$, HF: $20.7 \%$ ), fat (NF: $4.4 \%$, HF: $23.5 \%$ ), fiber (NF: $4.4 \%$, HF: 3.5\%), ash (NF: 7.0\%, HF: 5.5\%), nitrogen free extract (NF: 50.2\%, HF: 39.8\%), total calories (NF: $341 \mathrm{kcal} / 100 \mathrm{~g}$, HF: $460 \mathrm{kcal} / 100 \mathrm{~g}$ ).

The leaves of G. sylvestre were dried and extracted with $50 \%$ hydrous alcohol (at $60^{\circ} \mathrm{C}$ for $3 \mathrm{~h}$ ). It was then dried before being used as gymnema extract $(\mathrm{G})$ (total gymnemic acid content: ${ }^{14)} 2.4 \%$ ). Chitosan (C) employed here was adjusted to $85 \%$ deacetylation, $10^{5}-10^{6}$ molecular weight, and $100 \mathrm{cps}$ viscosity (estimated by a B-type viscosity meter at $0.5 \%$ and $20^{\circ} \mathrm{C}$ ).

Experimental Animals and Rearing Conditions Fiveweeks-old male Jcl: Wistar rats were obtained from Nihon Crea Co., Ltd. Two rats were kept in each polycarbonate cage 
(CL-0106 from Nihon Crea Co., Ltd.) with a size of $345 \times 403 \times 177 \mathrm{~mm}$, and were maintained at room temperature $\left(23 \pm 2{ }^{\circ} \mathrm{C}\right)$ and a relative humidity of $55 \pm 5 \%$ on a light/dark cycle of $14 \mathrm{~h} / 10 \mathrm{~h}$ (lighted up between 6:00 am and 8:00 pm). All rats were provided with NF for two weeks after initiation of preliminary rearing, and thereafter they were classified into NF-fed and HF-fed groups. The NF- and HFfed groups were provided with NF and HF respectively until the end of the experiment. Four weeks after classification into NF- and HF-fed groups, each of the two groups (11week-old rats) was divided into the following four groups (n: number of individuals reared in each group $=10)$ : control groups (cont-NF, cont-HF), G (G-orally-provided) groups (G-NF, G-HF), C (C-orally-provided) groups (C-NF, C-HF), and $\mathrm{GC}$ (provided with a mixture of $\mathrm{G}$ and $\mathrm{C}$ ) groups (GC$\mathrm{NF}$, GC-HF). The treated groups were orally administered with $\mathrm{G}, \mathrm{C}$ and $\mathrm{GC}$ once a day (10:00 a.m.-12:00 noon) for 10 weeks. The oral doses of G, C and GC were; G: $33 \mathrm{mg} / \mathrm{kg}$ (=amount required to stabilize blood glucose value in men), $\mathrm{C}: 8 \mathrm{mg} / \mathrm{kg}$ (=amount required to decrease plasma cholesterol value in men), and GC: $41 \mathrm{mg}$ ( $33 \mathrm{mg}$ of $\mathrm{G}+8 \mathrm{mg}$ of $\mathrm{C}) / \mathrm{kg}$ respectively. Each dose, after being suspended in $2 \mathrm{ml}$ of distilled water, was given through a stomach tube. On the other hand, $2 \mathrm{ml}$ of distilled water was orally given to each control group in the same way. Rats were allowed ad libitum to access the feed stuff and drinking water; body weight and feed intake was measured everyday during this experimental period. Rats were starved for $18 \mathrm{~h}$ after the end of the experiment, and blood was taken from the jugular veins under anesthesia using sodium pentobarbital. This blood was used for measurement of plasma lipid and hematological biochemical parameters. Heart, liver and kidney were extracted for measurement of their weight. In addition, the extracted livers were used for measurement of liver lipids and histopathlogical examination. Moreover, kidneys were also used for histopathological examination.

Hematological Biochemical Examination Immediately after blood samples were collected, the red blood cell (RBC) counts and white blood cell (WBC) counts were measured by the use of the Celltak (product of Nihon Koden Corp.). Thereafter, blood plasma was isolated and total cholesterol, high-density lipoprotein (HDL)-cholesterol, and triglyceride were measured by the use of Spotchem2-T-Chol, Spotchem2HDL-C and Spotchem2-TG (Arkray Co., Ltd.), respectively. Further measurements were carried out for blood urea nitrogen (BUN), albumin (Alb), creatinine (Cre), glutamic-oxaloacetic transaminase (GOT), glutamic-pyruvic transaminase (GPT) and total bilirubin (T-Bil) by the use of Spotchem2BUN, Spotchem2-Alb, Spotchem2-Cre, Spotchem2-GOT, Spotchem2-GPT and Spotchem2-T-Bil (Arkray Co., Ltd.), respectively. For quantification of these parameters, the Spotchem SP-4410 ${ }^{15)}$ (Arkray Co., Ltd.) was utilized. Furthermore, the free fatty acid (FFA) content and lipoperoxide activity (Lipop) were measured by use of the Iatrolipo NFFA (Yatoron Co., Ltd.) and Determiner LPO (Kyowa Medics Co., Ltd.), respectively.

Organ Weight and Liver Lipid The extracted organs (heart, liver and kidney) were perfused by the use of cooled physiological saline for removal of remaining blood. Thereafter, the organs were weighed and the livers were frozen at $-30{ }^{\circ} \mathrm{C}$ for preservation. Lipids were extracted from the liv- ers by the method of Folch et al. ${ }^{16)}$ and total cholesterol and triglyceride levels were measured by use of commercial assay kits (Determiner TC555: CE-COD-POD method, ${ }^{17)}$ Determiner TG.S : LPL-GK-GPO method $^{18)}$ ) (Kyowa Medics Co., Ltd.).

Histopathological Examination Extracted liver and kidney were stained with hematoxylin-eosin (HE) for histopathological examination, and the tissue samples were examined microscopically.

Statistical Treatment In each diet group, after performing one-way analysis of variance, the significance of a difference was tested by Dunnet's method and $p<0.05 v s$. control group was considered to be statistically significant. To evaluate the effect of co-administration of $\mathrm{G}$ and $\mathrm{C}$, the group receiving GC was compared with the control group and with the $\mathrm{C}$ group using Student's $t$-test. Administration of GC was considered to be significantly different from $\mathrm{C}$ when the group that received GC showed a greater effectiveness than both the $\mathrm{C}$ group and the control group at $p<0.05$.

\section{RESULTS}

Feed Intake and Body Weight In the HF-fed group, the feed intake tended to be lower by about $10 \%$ than the NF-fed group from the time feed was changed from NF to HF during the experimental period. Addtionally, the feed intake of the G-HF, C-HF and GC-HF groups almost the same as the contHF group during the experimental period. Similarly, in the NF-fed group, no significant difference was found in the feed intake of the G-NF, C-NF and GC-NF groups when compared to the cont-NF group (data not shown).

The changes in body weight in the NF- and HF-fed groups during the experimental period are shown in Fig. 1. The body weight in the cont-HF group tended to increase from the 5th week after the switch from NF-feeding to HF-feeding (the 7 th week from the start of feeding) to the end of the experiment. Nevertheless, in the G-HF, C-HF and GC-HF groups, the increase in body weight was repressed from the beginning of oral administration of G, C, GC to the end of the experiment. At the end of the experiment, the body weights of the G-HF, C-HF and GC-HF groups were significantly depressed when compared to cont-HF. In the NF-fed group, no significant difference was found in the increase of body weights among the G-NF, C-NF and GC-NF groups compared to cont-NF during the experimental period.

Plasma Lipid Plasma total cholesterol and triglyceride were measured after starvation (for $18 \mathrm{~h}$ ) at the end of the experiment. These values are shown in Fig. 2. In the NF-fed group, the plasma total cholesterol value in the G-NF group had no statistical significant difference compared to cont-NF. Nevertheless, the value in the C-NF group displayed a significant decrease $(p<0.05)$ compared to cont-NF. Also, in the GC-NF group, its value was significant decreased $(p<0.05)$ compared to cont-NF. In addition, in a comparison of the plasma total cholesterol between cont-NF and GC-NF, or between C-NF and GC-NF, a significant decrease $(p<0.05)$ was seen in both cases. In the HF-fed group, there were no significant differences between the plasma total cholesterol values in cont-HF and its value in G-HF, C-HF and GC-HF. In the NF-fed group, the plasma triglyceride value in the G-NF, C$\mathrm{NF}$ and GC-NF groups showed a significant decrease 

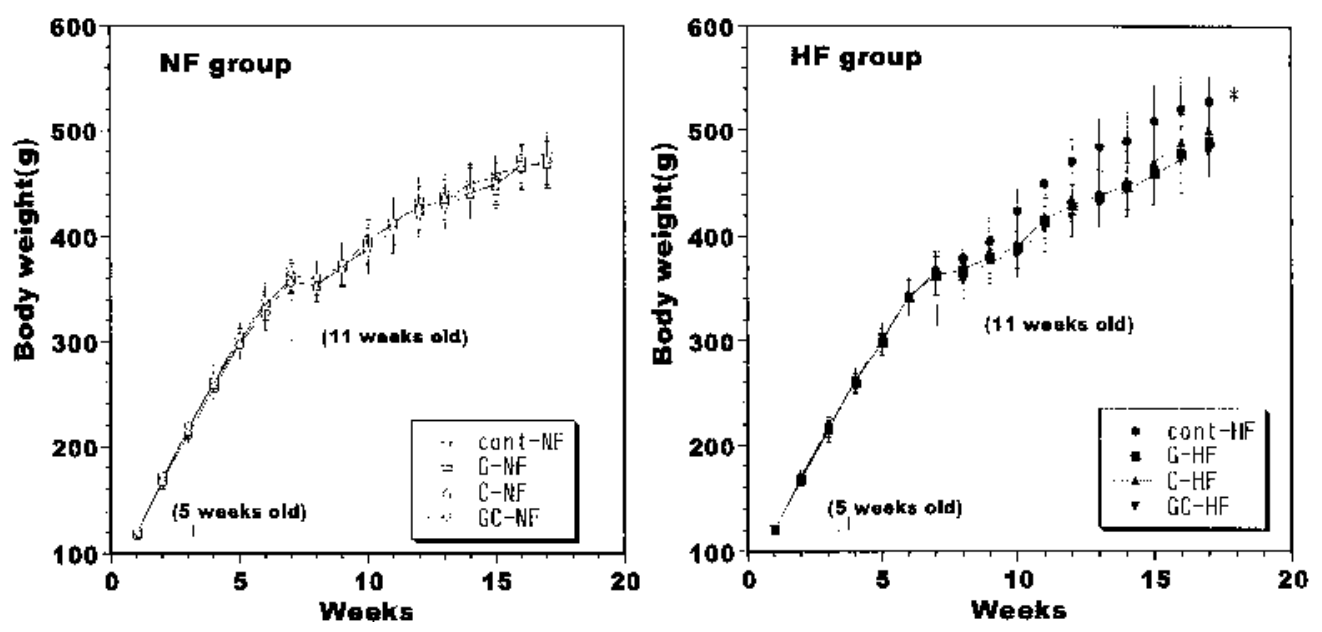

Fig. 1. Change of Body Weight in Rats Fed on NF or HF during the Experimental Period

Each point represents the mean of body weight. Vertical bars represent S.D. $(n=10)$. Symbol (*) represents $p<0.05$ compared with G or C or GC-HF at the end of the experiment.
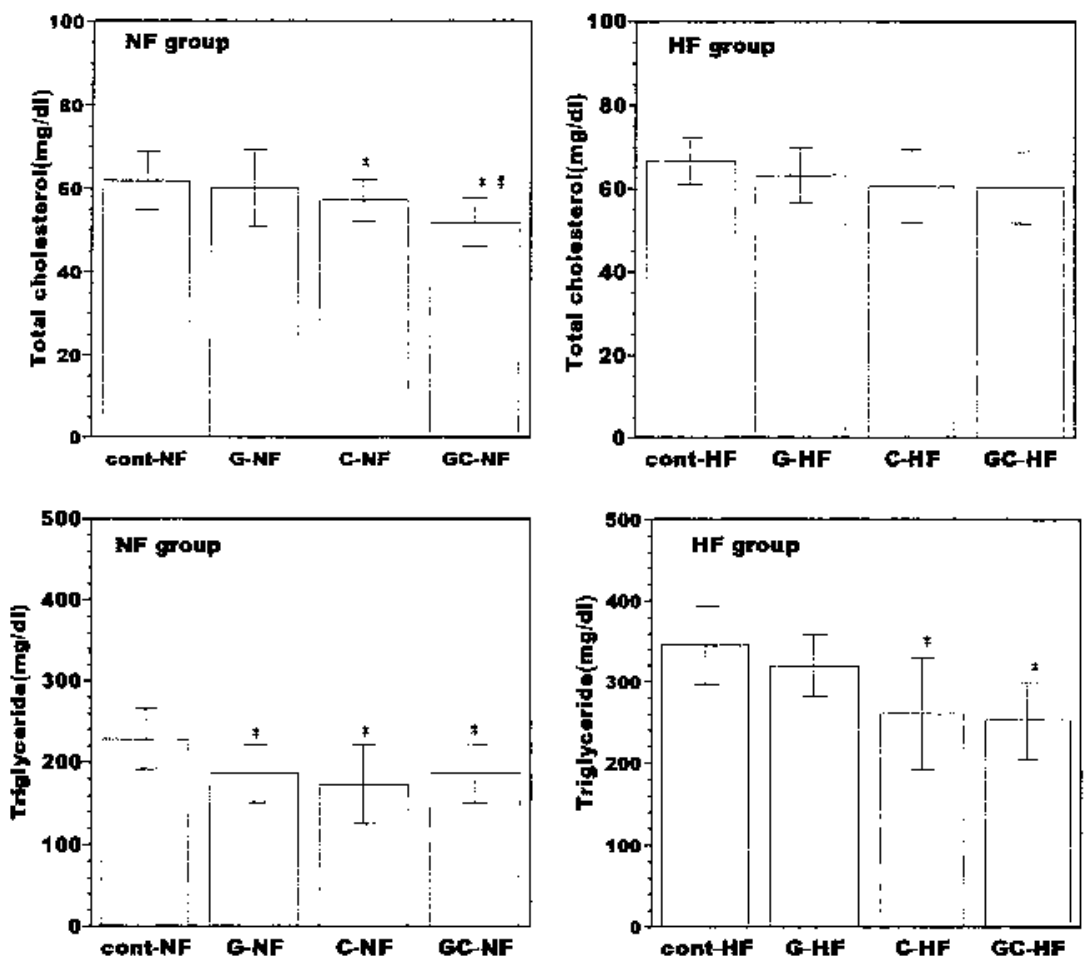

Fig. 2. Effects of Administration with G, C, GC on Plasma Lipid in Rats Fed on NF or HF at the End of the Experiment Vertical bars represent S.D. $(n=10)$. Statistical difference: $* p<0.05$ compared with cont-NF, $\# p<0.05$ compared with C-NF.

$(p<0.05)$ compared to cont-NF. In comparisons between cont-NF and GC-NF or between C-NF and GC-NF, no significant difference was seen in both cases and no effect of coadministration with $\mathrm{G}$ and $\mathrm{C}$ on the plasma triglyceride value was identified. In the HF-fed group, plasma triglyceride values in G-HF had a decreasing tendency $(p<0.1)$ compared to cont-HF, though not significant statistically. In the case of $\mathrm{C}$ HF or GC-HF, a significant decrease $(p<0.05)$ compared to cont-HF was noted. Moreover, no significant difference was found in a comparison of its value between $\mathrm{GC}-\mathrm{HF}$ and $\mathrm{C}$ HF. In addition, HDL-cholesterol levels were $26.8 \pm 4.9 \mathrm{mg} / \mathrm{dl}$ in cont-NF and $26.4 \pm 4.7 \mathrm{mg} / \mathrm{dl}$ in cont-HF. In both NF- and HF-fed groups, no influence was identified for $\mathrm{G}, \mathrm{C}$ and $\mathrm{GC}$ by oral administration compared to cont-NF or cont-HF (data not shown).

Hematological Biochemical Parameters The results of hematological biochemical examination carried out after starvation (for $18 \mathrm{~h}$ ) at the end of the experiment are shown in Table 1. In the NF-fed group, there was no influence of administration with $\mathrm{G}, \mathrm{C}, \mathrm{GC}$ on $\mathrm{RBC}$ count, WBC count, BUN, albumin, creatinine, GOT, GPT, total bilirubin, FFA and lipoperoxide compared to cont-NF. In addition, there was no effect in the HF-fed group administrated with $\mathrm{G}, \mathrm{C}, \mathrm{GC}$ compared to cont-HF.

Organ Weight and Liver Lipid The organ weights of rats are shown in Table 2. In both NF-fed and HF-fed groups, 

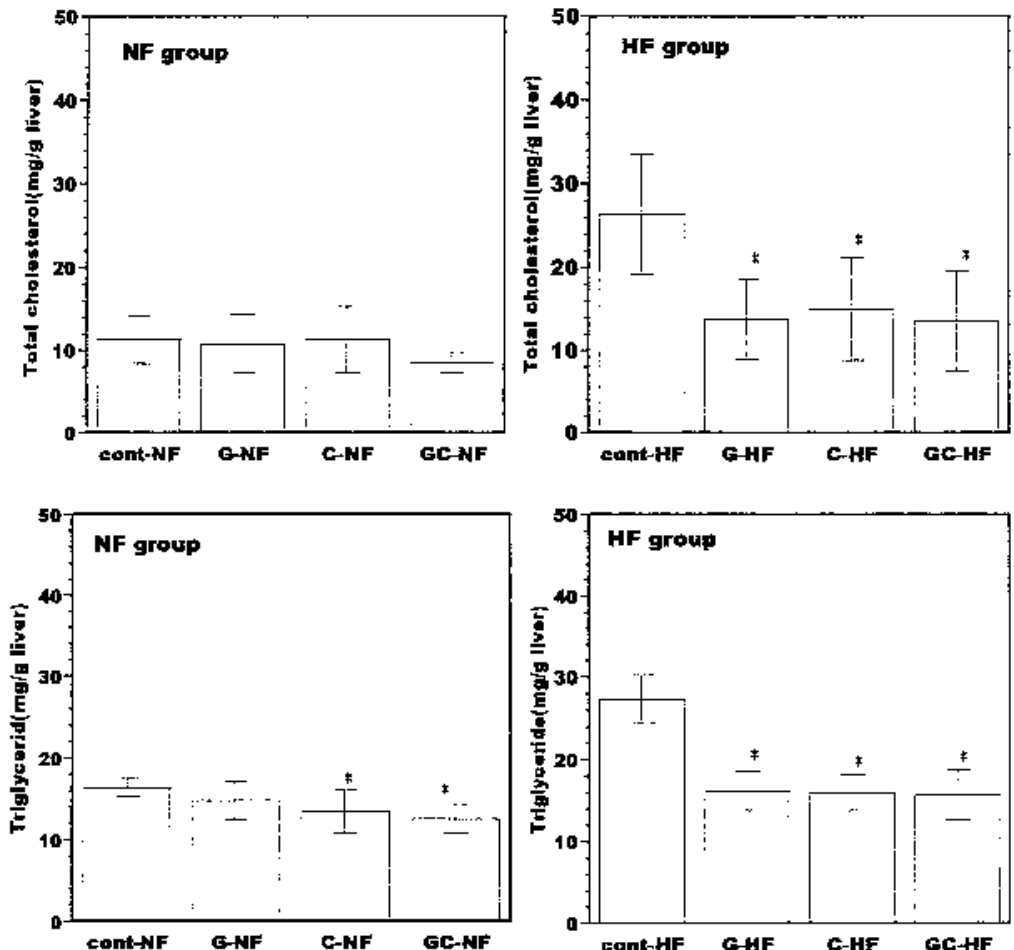

Fig. 3. Effects of Administration with G, C, GC on Liver Lipid in Rats Fed on NF or HF at the End of the Experiment

Vertical bars represent S.D. $(n=8)$. Statistical difference: $* p<0.05$ compared with control group.

Table 1. Results of Hematological and Plasma Biochemical Examination at the End of the Experiment

\begin{tabular}{|c|c|c|c|c|c|c|c|c|c|c|}
\hline & $\begin{array}{c}\mathrm{RBC} \\
\left(\times 10^{4} / \mathrm{mm}^{3}\right)\end{array}$ & $\begin{array}{c}\text { WBC } \\
\left(\times 10^{2} / \mathrm{mm}^{3}\right)\end{array}$ & $\begin{array}{l}\text { BUN } \\
(\mathrm{mg} / \mathrm{dl})\end{array}$ & $\begin{array}{l}\text { Alb } \\
(\mathrm{g} / \mathrm{dl})\end{array}$ & $\begin{array}{c}\text { Cre } \\
(\mathrm{mg} / \mathrm{dl})\end{array}$ & $\begin{array}{l}\text { GOT } \\
\text { (IU/1) }\end{array}$ & $\begin{array}{l}\text { GPT } \\
(\mathrm{IU} / \mathrm{l})\end{array}$ & $\begin{array}{c}\text { T-Bil } \\
(\mathrm{mg} / \mathrm{dl})\end{array}$ & $\begin{array}{c}\text { FFA } \\
(\mu \mathrm{eq} / 1)\end{array}$ & $\begin{array}{c}\text { Lipop } \\
(\mathrm{nmol} / \mathrm{ml})\end{array}$ \\
\hline Cont-NF & $979 \pm 109$ & $146 \pm 14$ & $29 \pm 4$ & $3.7 \pm 0.3$ & $0.6 \pm 0.2$ & $52.4 \pm 14.4$ & $22.0 \pm 6.1$ & $0.3 \pm 0.1$ & $500 \pm 141$ & $0.7 \pm 0.4$ \\
\hline G-NF & $973 \pm 86$ & $150 \pm 13$ & $29 \pm 2$ & $3.7 \pm 0.4$ & $0.8 \pm 0.3$ & $39.1 \pm 19.8$ & $20.2 \pm 8.6$ & $0.3 \pm 0.1$ & $490 \pm 129$ & $0.4 \pm 0.4$ \\
\hline C-NF & $982 \pm 90$ & $147 \pm 18$ & $29 \pm 3$ & $3.9 \pm 0.5$ & $0.9 \pm 0.3$ & $43.2 \pm 11.2$ & $23.2 \pm 5.9$ & $0.2 \pm 0.0$ & $482 \pm 110$ & $0.5 \pm 0.5$ \\
\hline GC-NF & $978 \pm 102$ & $152 \pm 12$ & $29 \pm 3$ & $3.8 \pm 0.3$ & $0.9 \pm 0.3$ & $48.1 \pm 14.8$ & $19.8 \pm 7.2$ & $0.2 \pm 0.1$ & $475 \pm 135$ & $0.4 \pm 0.3$ \\
\hline Cont-HF & $996 \pm 89$ & $140 \pm 20$ & $28 \pm 4$ & $3.8 \pm 0.2$ & $0.8 \pm 0.2$ & $39.1 \pm 10.5$ & $24.3 \pm 7.0$ & $0.3 \pm 0.2$ & $533 \pm 148$ & $0.5 \pm 0.3$ \\
\hline G-HF & $990 \pm 109$ & $149 \pm 28$ & $26 \pm 4$ & $3.7 \pm 0.3$ & $0.9 \pm 0.3$ & $46.8 \pm 12.8$ & $22.3 \pm 9.1$ & $0.3 \pm 0.2$ & $571 \pm 135$ & $0.4 \pm 0.4$ \\
\hline $\mathrm{C}-\mathrm{HF}$ & $965 \pm 97$ & $144 \pm 24$ & $27 \pm 5$ & $3.6 \pm 0.5$ & $0.9 \pm 0.3$ & $48.2 \pm 15.6$ & $20.8 \pm 5.0$ & $0.4 \pm 0.3$ & $560 \pm 111$ & $0.5 \pm 0.4$ \\
\hline GC-HF & $978 \pm 80$ & $138 \pm 21$ & $27 \pm 4$ & $3.7 \pm 0.3$ & $0.9 \pm 0.4$ & $41.4 \pm 16.0$ & $21.4 \pm 7.6$ & $0.3 \pm 0.2$ & $513 \pm 110$ & $0.5 \pm 0.5$ \\
\hline
\end{tabular}

Each value represents the mean \pm S.D. $(n=10)$.

Table 2. Organ (Heart Liver Kidney) Weight of Rats in the Experimental Groups at the End of the Experiment

\begin{tabular}{lccc}
\hline \hline & Heart $(\mathrm{g} / 100 \mathrm{~g})$ & Liver $(\mathrm{g} / 100 \mathrm{~g})$ & Kidney $(\mathrm{g} / 100 \mathrm{~g})$ \\
\hline Cont-NF & $0.30 \pm 0.02$ & $3.46 \pm 0.2$ & $0.59 \pm 0.06$ \\
G-NF & $0.29 \pm 0.02$ & $3.39 \pm 0.2$ & $0.59 \pm 0.04$ \\
C-NF & $0.30 \pm 0.02$ & $3.44 \pm 0.2$ & $0.60 \pm 0.02$ \\
GC-NF & $0.30 \pm 0.02$ & $3.57 \pm 0.3$ & $0.60 \pm 0.04$ \\
Cont-HF & $0.26 \pm 0.01$ & $3.02 \pm 0.2$ & $0.45 \pm 0.03$ \\
G-HF & $0.29 \pm 0.03$ & $3.39 \pm 0.4$ & $0.57 \pm 0.04$ \\
C-HF & $0.28 \pm 0.02$ & $3.28 \pm 0.2$ & $0.56 \pm 0.04$ \\
GC-HF & $0.29 \pm 0.02$ & $3.46 \pm 0.3$ & $0.58 \pm 0.04$ \\
\hline
\end{tabular}

Each value represent the mean \pm S.D. $(n=8)$.

no influence on organ weights was identified for oral administration of $\mathrm{G}, \mathrm{C}$ and $\mathrm{GC}$ compared to each control group. Total cholesterol and triglyceride levels in liver removed immediately after completion of the experiment are shown in Fig. 3. Total cholesterol values in the NF-fed group were not influenced by oral administration with G, C or GC compared to cont-NF. However, in the HF-fed group, the values in the G-HF, C-HF and GC-HF groups showed a significant decrease $(p<0.05)$ compared to cont-HF. In the NF-fed group, triglyceride values in G-NF had no significant difference compared to cont-NF, however, the C-NF and GC-NF groups showed significant decrease $(p<0.05)$ compared to cont-NF. Nevertheless, in the HF-fed group, tirglyceride values in the G-HF, C-HF and GC-HF groups had a significant decrease $(p<0.05)$ compared to cont-HF.

Histopathological Examination Rats in cont-HF and G-HF at the end of the experiment are shown in Fig. 4. Rat in the cont-HF group (left side) grew corpulent compared to rats in the G-HF group (right side). In addition, histopathological findings revealed that intraperitoneal fat was accumulated in the cont-HF group, while it was markedly inhibited in the G-HF group (Fig. 4). Nearly the same findings as G$\mathrm{HF}$ were noted in the $\mathrm{C}-\mathrm{HF}$ and GC-HF groups (data not shown). The results of histopathological examination of he- 

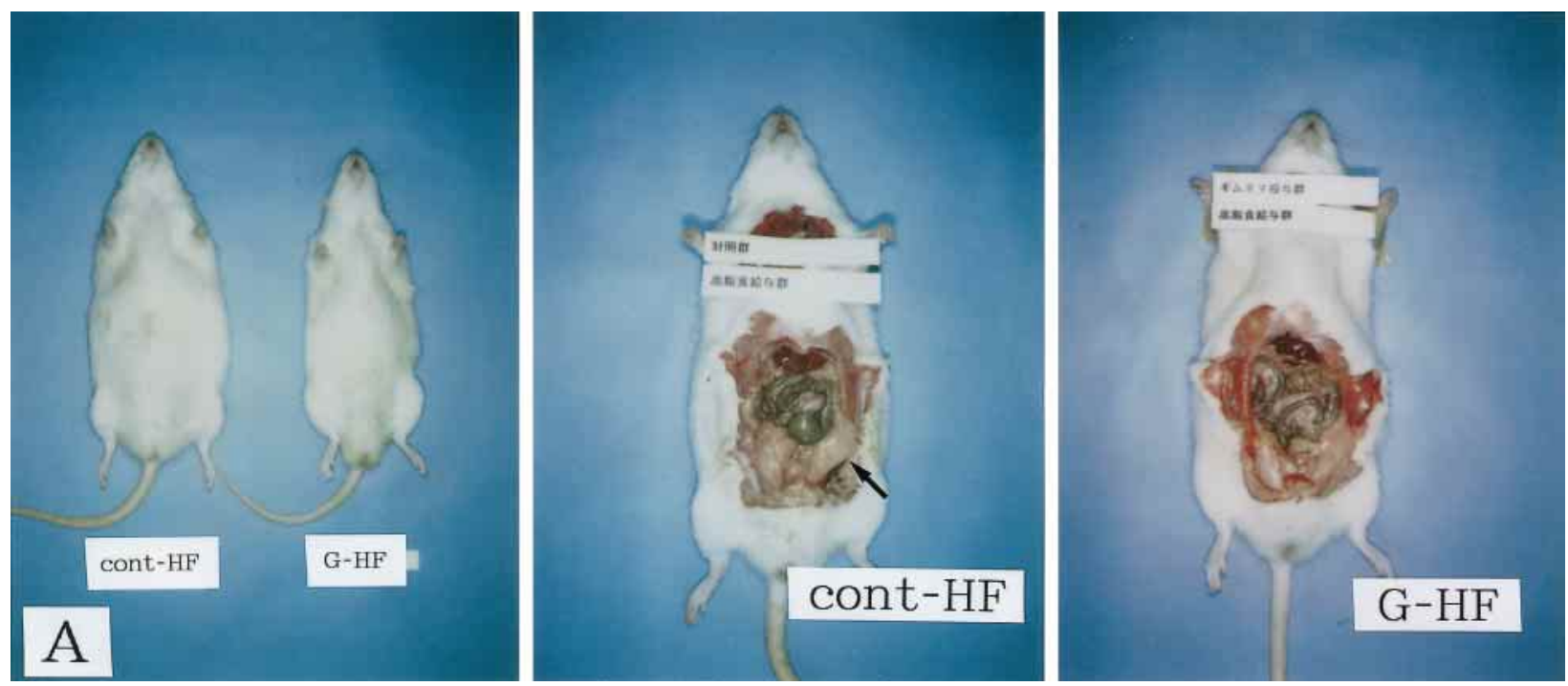

Fig. 4. Rats at the End of the Experiment

Left side, cont-HF; right side, G-HF; arrow, intraperitoneal fat.
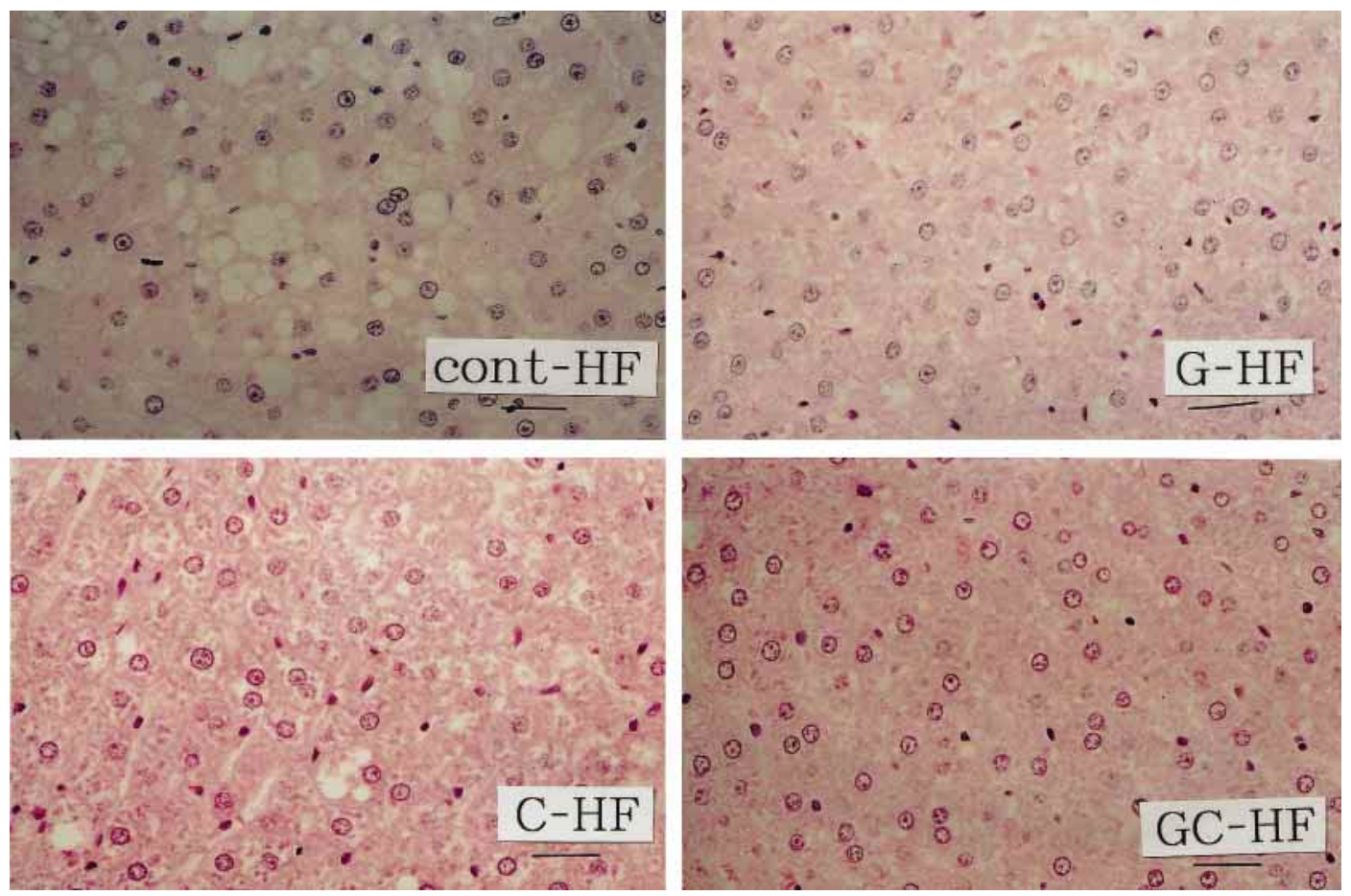

Fig. 5. Hepatocyte in Cont-HF, G-HF, C-HF and GC-HF

Hematoxylin-eosin stain $(\times 150)$.
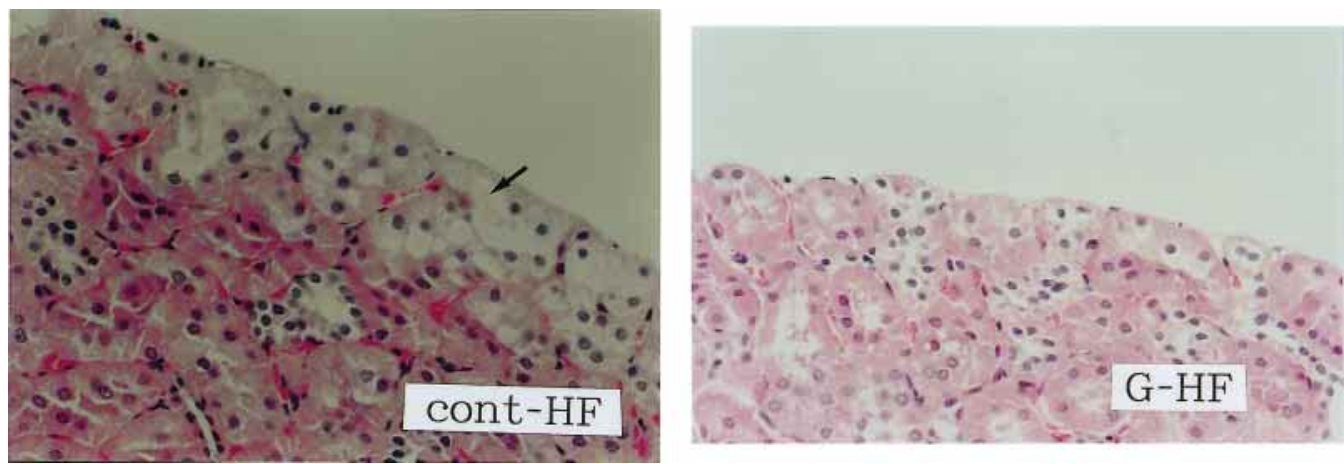

Fig. 6. Kidney in Cont-HF and G-HF

Hematoxylin-eosin stain $(\times 300)$. Arrow: fat droplet vacuoles. 
patocyte in the HF-fed groups are shown in Fig. 5. Fat droplets markedly accumulated in the liver of cont-HF, but fat deposition was inhibited in G-HF as a whole, though some fat vacuoles were evident. The same results were obtained with the $\mathrm{C}-\mathrm{HF}$ and $\mathrm{GC}-\mathrm{HF}$ groups. In the kidney, fat droplet vacuoles were noted on the epithelium of renal tubules in cont-HF, while they were noted only on the surface of cortex in G-HF (Fig. 6).

Nearly the same findings as G-HF were noted in the C-HF and GC-HF groups (data not shown). In addition, in NF fed groups, no abnormal influence was histopathologically observed (data not shown).

\section{DISCUSSION}

Since G. sylvestre has been widely used as dietary a food material in Japan, it has become more important to investigate the long-term effects of administration. Moreover, edible fats occupy an increasing portion of the total caloric intake in the Japanese diet of today. Therefore, the influence of $G$. sylvestre, which is effective in improving carbohydrate metabolism, on lipid metabolism in a high fat diet is very interesting from a real life point of view.

In this study, abnormal values were not observed upon hematological biochemical and histopathological examination. These results suggest the safety of $G$. sylvestre as a dietary food or functional food material. In cont-HF, the plasma cholesterol level was little affected by HF feeding, but plasma triglyceride levels and liver lipids (cholesterol and triglyceride) tended to be increased. Beef tallow has less influence on blood cholesterol level, but it increases the blood triglyceride level and accumulates liver lipid just like other edible fats. ${ }^{19,20)}$ These effects were reported by Pan et $a l$. and are similar to the plasma and liver lipids level in contHF.

Concerning plasma total cholesterol level, administration of $\mathrm{G}$ alone did not have any effect, although it was found in $\mathrm{NF}$-fed rats that the lowering effect of $\mathrm{C}$ was enhanced by coadministration of $\mathrm{G}$ and $\mathrm{C}$. The effect of chitosan on blood cholesterol by oral administration has previously reported. ${ }^{12,13)}$ Moreover, ascorbic acid is known to intensify the inhibitory effect of chitosan on lipid absorption in the digestive tract. This potentiating effect of ascorbic acid is thought to be due to the increased solubility of chitosan in the digestive tract and to the decreased viscosity of chitosan induced by ascorbic acid. ${ }^{12)}$ Whether the triterpene saponin in G can display such an action similiar to ascorbic acid is not clear, although $\mathrm{G}$ may have affected the behavior of $\mathrm{C}$ in the digestive tract when combined with $\mathrm{C}$ and $\mathrm{G}$ may enhance the lowering effect of $\mathrm{C}$ on plasma total cholesterol level consequently. In both NF- or HF-fed rats, the plasma HDL-cholesterol level was not influenced by G, C or GC. Thus, the decrease of plasma total cholesterol in NF-fed rats can be considered to be due to the decrease of LDL- and VLDL-cholesterol. In NF-fed rats, $\mathrm{G}$ decreased the plasma triglyceride level and the same decreasing effect was also found in the C$\mathrm{NF}$ and GC-NF groups. Therefore, $\mathrm{G}$ can be expected to be equally effective as chitosan under the conditions of a normal fat diet. In HF-fed rats, this value was little affected by $\mathrm{G}$ and significantly decreased by $\mathrm{C}$ and GC. It was considered from these findings that the decreasing effect of $\mathrm{G}$ on plasma triglyceride levels was weaker than that of chitosan with a high fat diet.

In this study, the lowering effects of $\mathrm{G}$ on cholesterol and triglyceride levels in liver were identified using a high fat diet and gave the same results as with chitosan and with combined use. The decreasing effect of $\mathrm{G}$ on lipid accumulation in liver was also identified by histopathological examination of hepatocytes and no abnormality was noted. Moreover, the decreasing effects of $\mathrm{G}$ on intraperitoneal fat and fat drop vacuoles on the epithelium of kidney noted in obese rats fed a high fat diet were identified. In addition, hematological biochemical findings also showed no abnormal data. Thus, it was suggested that $G$. sylvestre would safely suppress the accumulation of liver lipids and visceral fat at the same level as chitosan and improve obesity as a whole when administered for a long time under a high fat diet. In general, the decrease of triglyceride ${ }^{21)}$ in liver may result from (1) the suppression of lipoprotein and lipid synthesis in liver, (2) the acceleration of fatty acid oxidation in liver, (3) the acceleration of VLDL secretion from liver. In general, the decrease of cholesterol in liver may result from (1) the effects of transfer acceleration of cholesterol from liver to blood, (2) the inhibition of cholesterol synthesis in liver, (3) increased of cholesterol to bile acid, (4) the hydrolysis improvement of cholesterol ester in liver, and (5) suppression of absorption of cholesterol from intestine. Generally, the decrease of cholesterol ${ }^{22}$ in liver may lead to a decrease of VLDL synthesized in liver and secreted to the blood. On the other hand, the decrease of cholesterol in liver may induce activation of the LDL receptor and as a result, the intake of LDL from blood to liver increases. In addition, the decrease of cholesterol in liver may lead to a shortage of blood cholesterol. In this study, the decreasing effect of $\mathrm{C}$ on total cholesterol and triglyceride levels in plasma tended to be linked to the decrease of these materials in liver. $\mathrm{G}$ decreased the cholesterol and triglyceride levels in liver more markedly than in plasma. These results suggest that $\mathrm{G}$ may affect lipid metabolism through a different mechanism to $\mathrm{C}$.

The active ingredients contained in G. sylvestre should be investigated further in detail. Gymnemic acid has been investigated frequently in relation to carbohydrate metabolism. Regarding the relationship between lipid metabolism and gymnemic acid, it has been reported by Nakamura et al. ${ }^{11)}$ that when the extract containing $58 \%$ of gymnemic acid was administrered at doses of more than $1.0 \mathrm{~g} / \mathrm{kg}$, the excretion of acidic steroids and neutral steroid to feces were significantly increased and hepatic cholesterol was decreased, but blood cholesterol level was unaffected. ${ }^{11)}$ These results, where hepatic lipids are more liable to be affected by gymnemic acid than blood lipids, agree with the results of the present study. However, it has also been reported that feed intake was decreased and body weight gains were consequently depressed at the above doses. ${ }^{11)}$ In the present study, the total gymnemic acid content in $\mathrm{G}$ was $2.4 \%$ and the oral dose of $\mathrm{G}$ was $33 \mathrm{mg} / \mathrm{kg}$. Thus, both gymnemic acid and the extract amount were drastically less than in the other report. ${ }^{11)}$ Though we can not directly compare our results with the results in the earlier report, ${ }^{11)}$ due differences in the experimental diet and dose of gymnemic extract, our results do not necessarily indicate that only gymnemic acid influences lipid metabolism. Other substances besides gymnemic acid may have an influ- 
ence on lipid metabolism. In this study, the mechanism of the decreasing effect exerted by $\mathrm{G}$ is not clear and more research is required. Further studies are required to clarify the effect of $G$. sylvestre on lipid metabolism.

\section{REFERENCES}

1) Nadkarni A. K., "Indian Material Medica," Vol. 1, Popular Prakashan Pvt, Ltd., Bombay, 1982, pp. 596-599.

2) Mhaskar K. S., Caius J. F., Ind. Med. Res. Memories., 16, 2-75 (1930).

3) Kurihara K., "Taste Modifiers. Handbook of Sensory Physiology," Vol. IV Chemical Sense, Part 2, Beidler L. M., Springer-Verlag, Berlin, 1971, pp. 365-370.

4) Terasawa H., Miyoshi M., Imoto T., Yonago Acta Med., 37, 117-127 (1994).

5) Abe T., J. Yonago Med. Ass., 44, 198-209 (1993).

6) Hirata S., Terasawa H., Katou T., Imoto T., J. Yonago Med. Ass., 43, 397-404 (1992).

7) Hirai S., Abe T., Imoto T., J. Yonago Med. Ass., 43, 392-396 (1992).

8) Baskaran K., Kizr B., Ahamath K. Radha., Shanmugasundaram E. R. B., J. Ethnopharmacology, 30, 295-305 (1990).
9) Yoshikawa M., Murakami T., Kadota M., Li Y., Murakami N., Matsuda H., Chem. Pharm. Bull., 45, 1671-1676 (1997).

10) Ota M., Tonosaki K., Miwa K., Furuwatari T., Ariyoshi Y., Biopolymers, 39, 199-205 (1996).

11) Nakamura Y., Tsumura Y., Tonogai Y., Shibata T., J. Nutr., 129, 1214-1222 (1999).

12) Kanauchi O., Deuchi K., Imasato Y., Kobayashi E., Biosci. Biotech. Biochem., 58, 1617-1620 (1994).

13) Deuchi K., Kanauchi O., Imasato Y., Kobayashi E., Biosci. Biotech. Biochem., 58, 1613-1616 (1994).

14) Yokota T., Mizutani K., Okada K., Tanaka O., Nippon Shokuhin Kyogyo Gakkaishi, 41, 202-205 (1994) (in Japanese).

15) Shibata A., J. New Rem. \& Clin., 42, 114-123 (1993).

16) Folch J., Lees M., Stanley S. H., J. Biol. Chem., 266, 497-507 (1957).

17) Richmond W., Clin. Chem., 19, 1350 (1973).

18) Uwajima T., Methods in Enzymol., 89, 243 (1982).

19) Pan D. A., Storlien L. H., J. Nutr., 123, 512-519 (1993).

20) Shimomura Y., Tamura T., Suzuki M., J. Nutr., 120, 1291-1296 (1990).

21) Dietschy J. M., Wilson J. D., New Eng. J. Med., 282, 1179-1241 (1970).

22) Venkatese S., Ward R. J., Peters T. J., Biochem. Biophys. Acta, 960, $61-66$ (1988). 